\title{
SOME FUNDAMENTAL LEMMAS ON PROJECTIVE SCHEMES
}

\author{
BY \\ YOSHIKAZU NAKAI $\left({ }^{1}\right)$
}

In this paper we shall prove some fundamental results on invertible sheaves o $\mathbf{n}$ projective schemes. All of them are almost trivial when we are working in the worl $d$ of nonnilpotent geometry, but phenomena changes completely when we are dealing with general schemes. These topics were brought to our attention during the period of investigation on criterion of ample sheaves on projective schemes $\left({ }^{2}\right)$. We shall borrow notations and terminologies from Grothendieck's book, Eléments de géométrie algébrique, which will be cited as [G].

1. Let $A$ be a noetherian ring and let $X=\operatorname{Spec}(A)$. As usual, we shall denote by $\mathcal{O}_{X}$ (or $\left.\mathcal{O}\right)$ the structure sheaf of $X$. Let $\mathscr{K}$ be the sheaf on $X$ whose stalk $\mathscr{K}_{x}$ at $x \in X$ is the total quotient ring of $\mathcal{O}_{x}$. Let $K$ be the total quotient ring of $A$, then we have a natural homomorphism

$$
\pi: K \rightarrow \Gamma(X, \mathscr{K}) .
$$

$\pi$ is a monomorphism. In fact, let $\alpha=a / b$ be an element of $K$ where $a$ and $b$ are elements of $A$, and $b$ is not a zero divisor of $A$. Then, by definition

$$
\pi(\alpha)_{\mathfrak{p}}=\phi_{\mathfrak{p}}(a) / \phi_{\mathfrak{p}}(b)
$$

where $\mathfrak{p}$ is an element of $\operatorname{Spec}(A)$ and $\phi_{\mathfrak{p}}$ is a natural homomorphism $A \rightarrow A_{\mathfrak{p}}$. This is well defined since $\phi_{\mathfrak{p}}(b)$ is not a zero-divisor of $A_{\mathfrak{p}}$. Hence $\pi(\alpha)=0$ if and only if $\phi_{\mathfrak{p}}(a)=0$ for all $\mathfrak{p} \in X$. It implies the annihilator of $a$ is not contained in any prime ideal of $A$, i.e., $a=0$.

In the next place, we shall show that $\pi$ is an epimorphism. To prove this, we shall consider an affine scheme $Y=\operatorname{Spec}(K)$ and a morphism

$$
\lambda: Y \rightarrow X
$$

induced by the injection

$$
j: A \rightarrow K
$$

Received by the editors September 21, 1962 and, in revised form, November 20, 1962.

(1) This work is supported by the Air Force Office of Scientific Research of the Air Research and Development Command under Contract No. AF 49(638)-494.

(2) Y. Nakai, A criterion of an ample sheaf on a projective scheme, Amer. J. Math. 85 (1963), 14-26. This will be cited as [N]. 
Let $\mathfrak{P}$ be a prime ideal of $K$ and let us put $\mathfrak{p}=\mathfrak{P} \cap A$. Then $\mathfrak{p}$ is a prime ideal of zero in $A$ and we can see easily $K_{\mathfrak{\beta}}$ is the total quotient ring of $A_{\mathfrak{p}}\left({ }^{3}\right)$. Let $s$ be a section of $\Gamma(X, \mathscr{K})$. Then $\tilde{s}=s \circ \lambda$ defines a section of $\Gamma\left(Y, \mathcal{O}_{Y}\right)$. On the other hand, $\Gamma\left(Y, \mathcal{O}_{Y}\right)$ is canonically isomorphic to $K(\mathrm{G}-\mathrm{I}-1.3 .7)\left({ }^{4}\right)$. Hence we have a canonical homomorphism

$$
\tau: \Gamma(X, \mathscr{K}) \rightarrow K
$$

It is almost trivial that

$$
\tau \circ \pi=\text { identity }
$$

Hence $\tau$ is surjective.

In the next place we shall prove that $\tau$ is a monomorphism. Let $s \in \Gamma(X, \mathscr{K})$ and let $x \in X$ be an arbitrary point of $X$. We shall show that $\tau(s)=0$ implies $\mathrm{s}_{x}=0$. Let $\mathfrak{p}_{1}, \cdots, \mathfrak{p}_{t}$ be the prime divisors of 0 in $\mathcal{O}_{x}$. Then $\tau(s)=0$ implies, among others, that $s=0$ in $\left(\mathcal{O}_{x}\right)_{\mathfrak{p}_{i}}(i=1, \cdots, t)$, i.e., the annihilator of $s_{x}$ in $\mathcal{O}_{x}$ is not contained in any one of the prime ideals of 0 in $\mathcal{O}_{x}$. Hence $s_{x}$ is not contained in $\bigcup_{i} \mathfrak{p}_{i}\left({ }^{5}\right)$ and there exists a nonzero-divisor $t$ in $\mathcal{O}_{x}$ such that $t s_{x}=0$. Hence, $s_{x}=0$.

Thus we have the following

TheOREM 1. Let $A$ be a noetherian ring and $X=\operatorname{Spec}(A)$ and let $K$ be the total quotient ring of $A$. Let $\mathscr{K}$ be the sheaf of total quotient rings of structure sheaf $\mathcal{O}_{X}$. Then $\Gamma(X, \mathscr{K})$ is canonically isomorphic to $K$.

As an immediate consequence of Theorem 1 we have the

Corollary. Let $A$ be a noetherian ring and let $S$ be a multiplicatively closed set consisting of nonzero-divisors of $A$. Let $X=\operatorname{Spec}(A)$ and let $Y=\operatorname{Spec}\left(A_{S}\right)$ and let $\mathscr{K}_{X}$ and $\mathscr{K}_{Y}$ be sheaves of total quotient rings of the structure sheaves $\mathcal{O}_{X}$ and $\mathcal{O}_{Y}$, respectively. Then the natural homomorphism $\Gamma\left(X, \mathscr{K}_{X}\right) \rightarrow \Gamma\left(Y, \mathscr{K}_{Y}\right)$ is bijective.

The following two lemmas are useful in the sequel.

LEMMA 1. Let $V$ be a vector space over a field $k$ and let $V_{0}$ be the given subspace of $V$. Let $x, y$ be two elements of $V$ such that at least one of them is not contained in $V_{0}$. Then the vector space composed of points $(a, b)$ in $k^{2}$ such that $a x+b y \in V_{0}$ is at most of one dimension.

Proof. If one of $x, y$ is contained in $V_{0}$, the assertion is trivial. Assume that both of them are not contained in $V_{0}$. If there exists a vector $(a, b) \neq(0,0)$ in $k^{2}$

(3) In fact, we have $A_{\mathfrak{p}}=K_{\mathfrak{p}}$.

(4) This means $\S 1.3 .7$ of Chapter I in [G].

(5) Cf. Zariski-Samuel, Commutative algebra, Vol. I, Van Nostrand, Princeton, N. J., 1958, p. 215. 
such that $a x+b y \in V_{0}$, then any vector $\left(a^{\prime}, b^{\prime}\right)$ such that $a b^{\prime}-a^{\prime} b \neq 0$ satisfies the condition $a^{\prime} x+b^{\prime} y \notin V_{0}$. Q. E. D.

LEMMA 2. Let $V$ be a vector space over a field $k$ with infinitely many elements and let $U_{1}, U_{2}, \cdots, U_{n}$ be given subspaces of $V$. Let $W$ be a subspace of $V$ not contained in any one of $U_{\alpha}(\alpha=1,2, \cdots, n)$. Then $W$ is not contained in $\bigcup_{\alpha=1}^{n} U_{\alpha}$.

Proof. We shall use the induction on the number $n$ of given subspaces. The case $n=1$ is trivial. Assume the lemma holds in the case where the number of subspaces is $<n$. Let $w$ be an element of $W$ not contained in $U_{1}, U_{2}, \cdots, U_{n-1}$. If $w$ is not contained in $U_{n}$ we have nothing to prove. Hence assume that $w \in U_{n}$. Let $w^{\prime}$ be an element of $W$ not contained in $U_{n}$. Then there exists a finite number of 1-dimensional vector subspaces $E_{1}, \cdots, E_{m}$ in $k^{2}$ such that for any vector $(a, b)$ not contained in $E_{1}, \cdots, E_{m}$, we have $a w+b w^{\prime} \notin U_{\alpha}(\alpha=1, \cdots, n)$ by Lemma 1 . By assumption, $k$ is an infinite field, hence $k^{2} \neq E_{1} \cup E_{2} \cup \cdots \cup E_{m}$. This proves the lemma. Q.E.D.

2. Let $X$ be a projective scheme over a field $k$ with infinitely many elements and let $S$ be a homogeneous coordinate ring of $X$, i.e., $X=\operatorname{Proj}(S)$. We shall say that $S$ is an irrelevant coordinate ring if any element of $S_{+}$is a zero divisor of $S$. As is remarked elsewhere, when a projective scheme is given, we can find always a coordinate ring which is not irrelevant( $\left.{ }^{6}\right)$. In the following, we shall always assume that any homogeneous coordinate ring $S$ of a projective scheme is not irrelevant. Let $x_{0}, x_{1}, \cdots, x_{r}$ be elements of degree 1 in $S$ such that $S=k\left[x_{0}, x_{1}, \cdots, x_{r}\right]$. Then if $S$ is not irrelevant and if $k$ contains infinitely many elements, there exist linear combinations $y_{i}=\sum_{j=0}^{r} a_{i j} x_{j}, a_{i j} \in k$, such that $y^{\prime} \mathrm{s}$ are not zero divisors and $S=k\left[y_{0}, y_{1}, \cdots, y_{n}\right]$. Let $X_{\alpha}=D_{+}\left(y_{\alpha}\right)\left({ }^{7}\right)$ be an affine open subset of $X$ with affine ring $A_{\alpha}=S_{\left(y_{x}\right)}\left({ }^{8}\right)$. Then these affine rings are contained in one and the same ring $K$, where $K$ is the set of elements of the form $a / b$ with $a$ and $b$ homogeneous elements in $S$ of the same degree and $b$ is not a zero divisor of $S$. We shall call $K$ the function ring of $X$. $K$ is nothing other than the total quotient ring of $A_{\alpha}$ for any $\alpha$. Moreover, the intersection of two open sets $X_{\alpha} \cap X_{\beta}$ is also an affine open set with affine ring $A_{\alpha}\left[A_{\beta}\right]$, whose total quotient ring is also $K$.

THEOREM 2. Let $X$ be a projective scheme over a field $k$ with infinitely many elements and assume that the homogeneous coordinate ring of $X$ is not irrelevant. Let $K$ be the function ring of $X$, and $\mathscr{K}$ the sheaf of total quotient rings of the structure sheaf $\mathcal{O}_{X}$. Then $\Gamma(X, \mathscr{K})$ is canonically isomorphic to the function ring $K$.

$\left({ }^{6}\right)$ See $\$ 4$ of $[N]$.

( $\left.{ }^{7}\right) D_{+}(f)$ is a set of primes in Proj $(S)$ not containing the given homogeneous element $f$.

${ }^{8}$ ) If $f$ is a homogeneous element, $S_{(f)}$ is the subset of degree 0 in $S_{f}$. For the precise definition, see [G-II]. 
Proof. As we remarked above we can cover $X$ with affine open sets $X_{\alpha}$ with affine coordinate ring $A_{\alpha}$. They satisfy the conditions:

(1) Total quotient ring of each $A_{\alpha}$ is equal to the function $\operatorname{ring} K$ of $X$.

(2) For any two indices $\alpha, \beta$ the affine coordinate ring $A_{\alpha}\left[A_{\beta}\right]$ of $X_{\alpha} \cap X_{\beta}$ is a quotient ring of $A_{\alpha}$ (or $A_{\beta}$ ) with respect to a multiplicatively closed set not containing zero divisors.

Let $\pi$ be a natural homomorphism of $\Gamma(X, \mathscr{K})$ into the direct sum $\coprod_{\alpha=0}^{n}\left(X_{\alpha}, \mathscr{K} \mid X_{\alpha}\right) . \pi$ is clearly a monomorphism. Let $\pi_{\alpha}$ be the direct summand of $\pi$ and let $s_{\alpha}$ be an element of $\Gamma\left(X_{\alpha}, \mathscr{K} \mid X_{\alpha}\right)$ for $\alpha=0,1, \cdots, n$. Then $\coprod_{\alpha} s_{\alpha}$ is in $\operatorname{Im}(\pi)$ if and only if $s_{\alpha}\left|X_{\alpha} \cap X_{\beta}=s_{\beta}\right| X_{\alpha} \cap X_{\beta}$. Since the restriction map $\Gamma\left(X_{\alpha}, \mathscr{K} \mid X_{\alpha}\right) \rightarrow \Gamma\left(X_{\alpha} \cap X_{\beta}, \mathscr{K} \mid X_{\alpha} \cap X_{\beta}\right)$ is bijective by the corollary of Theorem 1 , this implies that $s_{\alpha}$ 's are represented by one and the same element $s$ of $K$. Thus we can define a bijective map between $\Gamma(X, \mathscr{K})$ and $K$, and the proof is completed. Q.E.D.

3. Proposition 1. Let $A$ be a noetherian ring and let $K$ be the total quotient ring of $A$. Let us put $X=\operatorname{Spec}(A)$ and $Y=\operatorname{Spec}(K)$. Let $\lambda$ be a morphism $Y \rightarrow X$ induced by the injection map $A \rightarrow K$. Let $U$ be an open subset of $X$ of the form $X_{f}, f \in A$. Then we have $\Gamma(U, \mathscr{K})=\Gamma\left(\lambda^{-1}(U), \mathcal{O}_{Y}\right)\left({ }^{9}\right)$ and $\Gamma\left(U, \mathscr{K}^{*}\right)=\Gamma\left(\lambda^{-1}(U), \mathcal{O}_{Y}^{*}\right)$, where ${ }^{*}$ denotes the sheaf of multiplicative groups composed of units.

Proof. $K_{f}$ is the total quotient ring of $A_{f}$ and $\lambda^{-1}(U)$ is isomorphic to $\operatorname{Spec}\left(K_{f}\right)$. Hence $\Gamma(U, \mathscr{K})=K_{f}=\Gamma\left(\lambda^{-1}(U), \mathcal{O}_{Y}\right)$ by Theorem 1 and consequently $\Gamma\left(U, \mathscr{K}^{*}\right)=K_{f}^{*}=\Gamma\left(\lambda^{-1}(U), \mathcal{O}_{Y}^{*}\right)$. Q.E.D.

Let $X$ be a projective scheme over a field $k$ and let $S$ be a homogeneous coordinate ring of $X$ which is not irrelevant and let $K$ be the function ring of $X$ over $k$. Let us put $Y=\operatorname{Spec}(K)$. Then we can define a natural morphism

$$
\lambda: Y \rightarrow X \text {. }
$$

Let $U=D_{+}(f)$ be an open subset of $X$ defined by a homogeneous element $f$ in $S$. The affine coordinate ring $A$ of $U$ is given by $S_{(f)}$, and the total quotient ring of $S_{(f)}$ is the quotient ring of $K$ with respect to the multiplicatively closed set $\left\{(f / g)^{n}, n=0,1,2, \cdots\right\}$, where $g$ is an arbitrary fixed homogeneous element of $S$ having the same degree as $f$ and is not a zero-divisor of $S$. Then we have $\Gamma(U, \mathscr{K})=\left\{\right.$ total quotient ring of $\left.S_{(f)}\right\}=K_{f / g}$. On the other hand $\lambda^{-1}(U)=\operatorname{Spec}\left(K_{f / g}\right)$. Hence $\Gamma(U, \mathscr{K})=\Gamma\left(\lambda^{-1}(U), \mathcal{O}_{Y}\right)$ and similarly $\Gamma\left(U, \mathscr{K}^{*}\right)$ $=\Gamma\left(\lambda^{-1}(U), \mathcal{O}_{Y}^{*}\right)$.

Proposition 2. Using the notations and assumptions as above, we have $\Gamma(U, \mathscr{K})=\Gamma\left(\lambda^{-1}(U), \mathcal{O}_{Y}\right)$ and $\Gamma\left(U, \mathscr{K}^{*}\right)=\Gamma\left(\lambda^{-1}(U), \mathcal{O}_{Y}^{*}\right)$.

$\left({ }^{9}\right)$ Strictly speaking, this should be written as $\Gamma(U, \mathscr{K} \mid U)=\Gamma\left(\lambda^{-1}(U), \mathcal{O}_{Y} \mid \lambda^{-1}(U)\right)$. We adopted this expression for the sake of simplicity since this will not cause any confusion. 
As is known, open sets of the form $D_{+}(f)$ form a basis of open sets of $X$. Hence we have immediately from Proposition 2 the following

TheOREM 3. Let $X, Y$ be as above. We have $H^{q}(X, \mathscr{K})=H^{q}\left(Y, \mathcal{O}_{Y}\right)$ and $H^{q}\left(X, \mathscr{K}^{*}\right)=H^{q}\left(Y, \mathcal{O}_{Y}^{*}\right)$.

Let $X$ be a projective scheme and let $S$ be a nonirrelevant coordinate ring of $X$. Let $\mathfrak{p}_{1}, \mathfrak{p}_{2}, \cdots, \mathfrak{p}_{t}$ be all prime ideals of 0 in $S$. By our assumption, $\mathfrak{p}$ 's are in $\operatorname{Proj}(S)=X$. An open set $U$ containing all prime ideals $\mathfrak{p}_{1}, \cdots, \mathfrak{p}_{t}$ of zero will be called a general open subset of $S$.

Proposition 3. Let $U$ be a general open subset of $X$ which is of the form $D_{+}(f)$. Then we have

(i) $X-U$ contains no nonempty open set.

(ii) Let $V$ be any affine open set of $X$ of the form $D_{+}(g)$. Then the injection $i: \Gamma(V, \mathscr{K}) \rightarrow \Gamma(V \cap U, \mathscr{K})$ is an isomorphism.

Proof. Assume that $X-U$ contains a nonempty open set $V$. Then $V$ must contain at least one $\mathfrak{p} \in X$. Hence $V$ must contain all generalizations of $\mathfrak{p}$, in particular at least one of $\mathfrak{p}_{1}, \cdots, \mathfrak{p}_{t}$. It is a contradiction, proving the first assertion. As to the second assertion, we have by Proposition 2

$$
\Gamma(V, \mathscr{K})=\Gamma\left(\lambda^{-1}(V), \mathcal{O}_{Y}\right)=\Gamma\left(\lambda^{-1}(V \cap U), \mathcal{O}_{Y}\right)=\Gamma(V \cap U, \mathscr{K}),
$$

since $\lambda^{-1}(U)=Y$. Q.E.D.

4. Let $X$ be a projective scheme defined over a field $k$ and let $\mathcal{O}$ be the structure sheaf of $X$. Let $\mathscr{K}$ be as before the sheaf of total quotient rings of $\mathcal{O}$. Let us denote by $\mathcal{O}^{*}, \mathscr{K}^{*}$ the multiplicative groups of units in $\mathcal{O}$ and $\mathscr{K}$, respectively. Then we have an exact sequence

$$
\text { (1) } \rightarrow \mathcal{O}^{*} \rightarrow \mathscr{K}^{*} \rightarrow \mathscr{K}^{*} / \mathcal{O}^{*} \rightarrow(1)
$$

From this we have an exact sequence of cohomomology groups

$$
(1) \rightarrow H^{0}\left(X, \mathcal{O}^{*}\right) \rightarrow H^{0}\left(X, \mathscr{K}^{*}\right) \rightarrow H^{0}\left(X, \mathscr{K}^{*} / \mathcal{O}^{*}\right) \stackrel{\delta}{\rightarrow} H^{1}\left(X, \mathcal{O}^{*}\right) \rightarrow H^{1}\left(X, \mathscr{K}^{*}\right) .
$$

An element of $H^{0}\left(X, \mathscr{K}^{*} / \mathcal{O}^{*}\right)$ defines an invertible sheaf which is a subsheaf of $\mathscr{K}$. We shall call such a sheaf, following P. Cartier, a divisor of $X$. When $X$ is a reduced scheme it is easy to see that $H^{1}\left(X, \mathscr{K}^{*}\right)=\{0\}$ and the connecting homomorphism $\delta$ is necessarily surjective. In the general case, $H^{1}\left(X, \mathscr{K}^{*}\right)$ cannot be expected to vanish, though no counter example has been found yet. We shall prove in this section that if $k$ contains infinitely many elements then $\delta$ is always surjective.

This implies simply that any invertible sheaf is represented by a divisor since an element of $H^{1}\left(X, \mathcal{O}^{*}\right)$ corresponds in a 1-1 way to an isomorphism class of invertible sheaves on $X$. 
Lemma 3. Let $A$ be a noetherian ring and let $M$ be a finite A-module. Let $\mathrm{m}_{1}, \mathrm{~m}_{2}, \cdots, \mathrm{m}_{n}$ be maximal ideals of $A$ and assume that each $M \otimes A_{\mathfrak{m}_{i}}$ is a free $A_{\mathfrak{m}_{i}}$-module of rank $1(i=1,2, \cdots, n)$. Then there exists an element $f$ in $M$ such that $f \otimes 1$ is a free base of $M \otimes A_{\mathfrak{m}_{i}}$ for each $i$.

Proof. Let $f_{i}$ be an element of $M$ such that $f_{i} \otimes 1$ is a base of $M \otimes_{A} A_{\mathfrak{m}_{i}}$ over $A_{\mathrm{m}_{i}}$. We can assume without loss of generality that $f_{i}$ is contained in $\mathrm{m}_{j} M(j \neq i)$ since we can multiply $f_{i}$ by an element not contained in $m_{i}$ without changing the property that $f_{i} \otimes 1$ is a free base of $M \otimes A_{\mathfrak{m}_{i}}$. Let us put $f=f_{1}+f_{2}+\cdots+f_{n}$. Then $f$ will satisfy our requirement. In fact, we have $f \equiv f_{i}\left(\bmod m_{i} M\right)$ hence $M \otimes A_{\mathfrak{m}_{i}}=A_{\mathfrak{m}_{i}}(f \otimes 1)+\mathfrak{m}_{i}\left(M \otimes A_{\mathfrak{m}_{i}}\right)$. By our assumption $M \otimes A_{\mathfrak{m}_{i}}$ is a finite $A_{\mathfrak{m}_{i}}$-module. Then we have $M \otimes A_{\mathfrak{m}_{i}}=A_{\mathfrak{m}_{i}}(f \otimes 1)$.

Proposition 4. Let $X=\operatorname{Spec}(A)$ and assume that $A$ is an affine ring over an infinite field $k$. Then under the same assumptions and notations as in Lemma 3, there exists an open subset $U$ of $X$ containing all $\mathrm{m}_{\alpha}$ such that for any $x \in U$, we have $M_{x}=M \otimes A_{x}=A_{x}(f \otimes 1)$.

Proof. Let $M=A g_{1}+\cdots+A g_{t}$. Then by Lemma 3 there exists for each $\alpha$ $(\alpha=1, \cdots, n)$ and $i(i=1,2, \cdots, t)$ an element $s_{\alpha i}$ in $A$ not contained in $\mathrm{m}_{\alpha}$ such that we have

$$
s_{\alpha i} g_{i}=a_{i} f,
$$

where $a$ 's are elements of $A$. The set of elements satisfying the conditions $s g_{i} \in A f$ form a vector space over $k$ and it is not contained in any one of $m_{\alpha}(\alpha=1, \cdots, n)$. Hence there exists, by Lemma 2, an element $s_{i}$ in $A$ such that $s_{i} g_{i} \in A f$ and $s_{i} \notin \mathfrak{m}_{\alpha}(\alpha=1,2, \cdots, n)$. Let $s=s_{1} s_{2} \cdots s_{n}$. Then the set of prime ideals $\mathfrak{p}$ such that $\mathfrak{p} \not s$ form an open set $U$ and $U$ satisfies the requirement of the proposition.

THEOREM 4. Let $X$ be a projective scheme over a field $k$ with infinitely many elements. Let $\mathcal{O}$ be the structure sheaf of $X$ and let $\mathscr{K}$ be the sheaf of total quotient rings of $\mathcal{O}$. Let $\mathcal{O}^{*}, \mathscr{K}^{*}$ be the sheaves of multiplicative groups of units in $\mathcal{O}$ and $\mathscr{K}$, respectively. Then the connecting homomorphism

$$
\delta: H^{0}\left(X, \mathscr{K}^{*} / \mathcal{O}^{*}\right) \rightarrow H^{1}\left(X, \mathcal{O}^{*}\right)
$$

is surjective.

Proof. Let $X=\operatorname{Proj}(S)$ where $S$ is a nonirrelevant homogeneous coordinate ring of $X$ and let $\mathfrak{p}_{1}, \cdots, \mathfrak{p}_{n}$ be all the prime ideals of zero in $S$. Let $\mathrm{m}_{1}, \mathrm{~m}_{2}, \cdots, \mathrm{m}_{n}$ be fixed maximal ideals of $S_{+}$containing $\mathfrak{p}_{1}, \mathfrak{p}_{2}, \cdots, \mathfrak{p}_{n}$, respectively. Let $h$ be a linear form in $S$ such that $h \notin \mathrm{m}_{\alpha}\left({ }^{10}\right)(\alpha=1,2, \cdots, n)$ and let us put $X_{1}=D_{+}(h)$. Let $\mathscr{L}$ be an invertible sheaf. Then since $\mathscr{L} \mid X_{1}$ is a coherent sheaf, $M=\Gamma\left(X_{1}, \mathscr{L}\right)$ is a finite module over $A=\Gamma\left(X_{1}, \mathcal{O}\right)$ and $\mathscr{L} \mid X_{1}=\tilde{M}$. By Proposition 4, there

(10) The existence of such an element $h$ is assured by Lemma 2 . 
exists an affine open subset $U$ of $X_{1}$ containing all $\mathrm{m}_{\alpha}(\alpha=1,2, \cdots, n)$ such that there exists an element $f$ in $M$ such that $\mathscr{L}_{x}=\mathcal{O}_{x} f$ for any $x \in U$. Then $U$ is a general open subset and from the proof of Proposition 4 we can choose $U$ in such a way that $U$ satisfies the conditions of Proposition 3.

In the first place, we shall define an isomorphism of $\mathscr{L}$ and $\mathcal{O}$ in $U$ by transforming $f$ to 1 . Let $x_{1}$ be a point of $X$ not contained in $U$. Then there exists an affine open set $V_{1}$ containing the point $x_{1}$ and an element $f_{1}$ in $\Gamma\left(V_{1}, \mathscr{L}\right)$ such that $\mathscr{L}_{x}=\mathcal{O}_{x} f_{1}$ for any $x \in V_{1}$. In $U \cap V_{1}$, we have $\mathscr{L}_{x}=\mathcal{O}_{x} f_{1}=\mathcal{O}_{x} f$ and hence $f / f_{1}$ is a unit in $U \cap V_{1}$; i.e., $f / f_{1}$ is a section of $\Gamma\left(U \cap V_{1}, \mathcal{O}^{*}\right)$. By Proposition 3, we can extend this section to a section $a_{1}$ of $\Gamma\left(V_{1}, \mathscr{K}^{*}\right)$ in a unique way.

If $U \cup V_{1}$ does not cover $X$, take a point $x_{2}$ not contained in $U \cup V_{1}$. Then we can find an affine open set $V_{2}$ containing $x_{2}$, a section $f_{2}$ in $\Gamma\left(V_{2}, \mathcal{O}\right)$ such that $f / f_{2}$ is in $\Gamma\left(U \cap V_{2}, \mathcal{O}^{*}\right)$ and a section $a_{2}$ of $\Gamma\left(V_{2}, \mathscr{K}^{*}\right)$ which is the unique extension of $f / f_{2}$. We proceed with this process and finally we find a finite affine open covering $V_{1}, V_{2}, \cdots, V_{q}$ and sections $a_{1}, \cdots, a_{q}$ on $V_{1}, \cdots, V_{q}$ with coefficients in $\mathscr{K}^{*}$. We shall show that $a_{i} a_{j}^{-1}$ is a unit in $V_{i} \cap V_{j}$. For the sake of simplicity we take $i=1, j=2$. Then in the first place, $f_{1} / f_{2}=a_{12}^{\prime}$ is a unit in $V_{1} \cap V_{2}$. Moreover, $a_{12}^{\prime} \mid U \cap V_{1} \cap V_{2}=a_{1} a_{2}^{-1}$ by the definition of $a_{1}, a_{2}$. Again using the isomorphism of $\Gamma\left(V_{1} \cap V_{2}, \mathscr{K}\right) \rightarrow\left(V_{1} \cap V_{2} \cap U, \mathscr{K}\right)$ we see that $a_{12}^{\prime}=a_{1} a_{2}^{-1}$ in $V_{1} \cap V_{2}$, i.e., $a_{1} a_{2}^{-1}$ must be a unit in $V_{1} \cap V_{2}$. The open covering $\left\{U, V_{1}, V_{2}, \cdots, V_{q}\right\}$ and the sections $\left\{1, a_{1}, a_{2}, \cdots, a_{q}\right\}$ defines a divisor $\hat{\mathscr{L}}$ which is isomorphic to $\mathscr{L}$. Q.E.D.

HARVARD UNIVERSITY, Cambridge, Massachusetts

HiRoshima UNIVERSITY,

HiROSHIMA, JAPAN 